\title{
Hadronic parity violation and effective field theory
}

\author{
B. R. Holstein
}

University of Massachusetts - Amherst, MA, USA

ricevuto il 7 Ottobre 2011; approvato il 5 Maggio 2012

pubblicato online il 4 Luglio 2012

Summary. - The subject of hadronic parity violation is briefly reviewed and an approach based on effective field theory is advocated in order to resolve current problems.

PACS 21.30.Fe - Forces in hadronic systems and effective interactions.

PACS 23.20.-g - Electromagnetic transitions.

PACS 25.40. Cm - Elastic proton scattering.

PACS 25.20.-x - Photonuclear reactions.

\section{1. - Introduction}

After the 1956 suggestion for the existence of parity violation (PV) in the weak interaction by Lee and Yang and its experimental discovery in ${ }^{60}$ Co beta decay by Ambler et al., it was natural for experimenters to seek evidence for this phenomena in other situations, including strong and electromagnetic hadronic systems. The first to do so was Tanner who in 1958 looked for evidence in the ${ }^{19} \mathrm{~F}(p, \alpha){ }^{16} \mathrm{O}$ reaction [1]. Although the sensitivity of this experiment was not high enough to observe a signal, this was merely the first of a series of such experiments which have continued to the present time, the goal of which is to understand the small weak interaction effects in strong and electromagnetic systems. That the phenomenon exists is clear from experiments such as the $2 \%$ photon asymmetry in the electromagnetic decay of an isomer of ${ }^{180} \mathrm{Hf}[2]$

$$
A_{\gamma}\left({ }^{180} \mathrm{Hf}^{*} \rightarrow{ }^{180} \mathrm{Hf}+\gamma\right)=-(1.66 \pm 0.18) \times 10^{-2}
$$

or the nearly $10 \%$ asymmetry in the scattering of longitudinally polarized neutrons from ${ }^{139} \mathrm{La}[3]$

$$
A_{h}\left(\vec{n}+{ }^{139} \mathrm{La}\right)=(9.55 \pm 0.35) \times 10^{-2} .
$$

These effects are anomalously large and are allowed only by the amplification which arises due to the presence of nearly degenerate nuclear states which have the same spin 
TABLE I. - Weak NNM couplings as calculated in refs. [4,6,7]. All numbers are quoted in units of the "sum rule" value $S_{R}=3.8 \cdot 10^{-8}$.

\begin{tabular}{|c|c|c|c|c|}
\hline Coupling & $\begin{array}{c}\text { DDH [4] } \\
\text { Reasonable Range }\end{array}$ & $\begin{array}{c}\text { DDH [4] } \\
\text { "Best" Value }\end{array}$ & DZ [6] & FCDH [7] \\
\hline$f_{\pi}$ & $0 \rightarrow 30$ & +12 & +3 & +7 \\
$h_{\rho}^{0}$ & $30 \rightarrow-81$ & -30 & -22 & -10 \\
$h_{\rho}^{1}$ & $-1 \rightarrow 0$ & -0.5 & +1 & -1 \\
$h_{\rho}^{2}$ & $-20 \rightarrow-29$ & -25 & -18 & -18 \\
$h_{\omega}^{0}$ & $15 \rightarrow-27$ & -5 & -10 & -13 \\
$h_{\omega}^{1}$ & $-5 \rightarrow-2$ & -3 & -6 & -6 \\
\hline
\end{tabular}

but opposite parity. Indeed the natural scale of hadronic PV effects is $\sim G_{F} m_{\pi}^{2} \sim 10^{-7}$. In the fifty years since the discovery of parity violation, there have been a series of experiments conducted, both with and without the use of nuclear amplification, and a substantial data base now exists.

On the theoretical side, for the past thirty years it has became standard practice to analyze such experiments in terms of the meson-exchange model posited by Desplanques, Donoghue, and Holstein (DDH) in 1980 [4]. In this picture, the mesons are limited to $\pi^{ \pm}, \rho, \omega$ whose strong interactions are described via the Hamiltonian

$$
\begin{aligned}
\mathcal{H}_{\mathrm{st}}= & i g_{\pi N N} \bar{N} \gamma_{5} \tau \cdot \pi N+g_{\rho} \bar{N}\left(\gamma_{\mu}+i \frac{\mu_{V}}{2 M} \sigma_{\mu \nu} k^{\nu}\right) \tau \cdot \rho^{\mu} N \\
& +g_{\omega} \bar{N}\left(\gamma_{\mu}+i \frac{\mu_{S}}{2 M} \sigma_{\mu \nu} k^{\nu}\right) \omega^{\mu} N
\end{aligned}
$$

Typical — though not universally accepted - values are $g_{\pi N N}^{2} / 4 \pi \simeq 13.5$ and $g_{\rho}^{2} / 4 \pi=$ $\frac{1}{9} g_{\omega}^{2} / 4 \pi \simeq 0.67$ and, with the use of vector dominance to connect with the electromagnetic interaction, $\chi_{\rho}=\kappa_{p}-\kappa_{n}=3.7$ and $\chi_{\omega}=\kappa_{p}+\kappa_{n}=-0.12$.

For the weak interaction couplings we write a general form in terms of seven phenomenological couplings

$$
\begin{aligned}
\mathcal{H}_{\mathrm{wk}}= & \frac{f_{\pi}}{\sqrt{2}} \bar{N}(\tau \times \pi)_{z} N \\
& +\bar{N}\left(h_{\rho}^{0} \tau \cdot \rho^{\mu}+h_{\rho}^{1} \rho_{z}^{\mu}+\frac{h_{\rho}^{2}}{2 \sqrt{6}}\left(3 \tau_{z} \rho_{z}^{\mu}-\tau \cdot \rho^{\mu}\right)\right) \gamma_{\mu} \gamma_{5} N \\
& +\bar{N}\left(h_{\omega}^{0} \omega^{\mu}+h_{\omega}^{1} \tau_{z} \omega^{\mu}\right) \gamma_{\mu} \gamma_{5} N-h_{\rho}^{\prime 1} \bar{N}\left(\tau \times \rho^{\mu}\right)_{z} \frac{\sigma_{\mu \nu} k^{\nu}}{2 m_{N}} \gamma_{5} N
\end{aligned}
$$

where Barton's theorem has been used to eliminate CP-conserving couplings to the neutral pseudoscalar mesons $-\pi^{0}, \eta^{0}, \eta^{0^{\prime}}[5]$. Using quark model methods DDH tried to estimate values for these weak parameters and came up with reasonable ranges and best values for each, as shown in table I. Analysis of existing experiments in terms of these unknown weak couplings has produced the well known graph shown in fig. 1. 


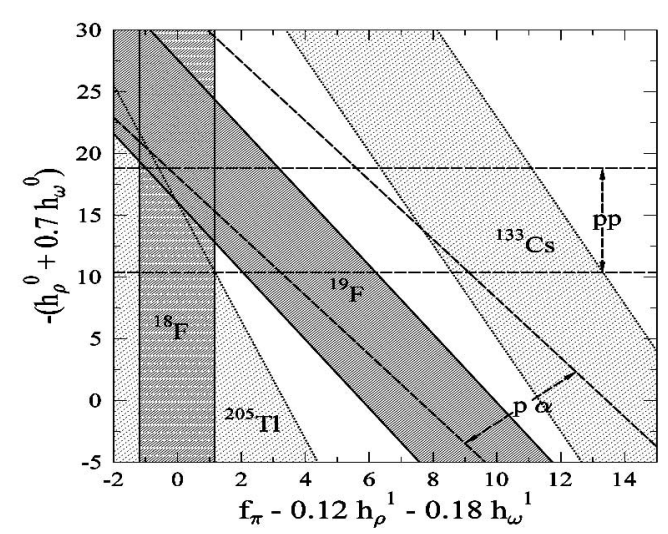

Fig. 1. - Nonrelativistic Feynman diagrams describing Compton scattering from a charged particle.

There is an obvious problem here and recently it was proposed by a number of groups to try to resolve this situation by use of effective field theory in order to remove the model dependence of the DDH potential, as well as the use of only experiments involving systems with $A<4$ in order to remove nuclear physics uncertainties. In the EFT picture there are at low energies only five low energy amplitudes which represent the five possible ways in which two nucleons can interact via S-P mixing:

i) $d_{t}(k)$ representing ${ }^{3} S_{1^{-}}{ }^{1} P_{1}$ mixing with $\Delta I=0$;

ii) $d_{s}^{0,1,2}(k)$ representing ${ }^{1} S_{0^{-}}{ }^{3} P_{0}$ mixing with $\Delta I=0,1,2$ respectively;

iii) $c_{t}(k)$ representing ${ }^{3} S_{1^{-}}{ }^{3} P_{1}$ mixing with $\Delta I=1$.

Unitarity requires that the phase of each amplitude be the sum of the $S$ - and $P$-wave amplitudes for each partial wave. Since the $S$-wave should be much larger than its $P$-wave counterpart, Danilov has suggested parameterization in terms of the representation [8]

$$
\lim _{k \rightarrow 0} c_{t}(k), d_{s}(k), d_{t}(k) \equiv \rho_{t} a_{t}, \lambda_{s}^{i} a_{s}, \lambda_{t} a_{t}
$$

where $a_{t}, a_{s}$ are the ${ }^{3} S_{1},{ }^{1} S_{0}$ scattering lengths. We need now five independent low energy and low A experiments in order to determine the five Danilov parameters and each experiment should be analyzed by theorists in terms of these LET's. Four such experiments have already been performed-

i) Longitudinally polarized proton scattering asymmetry from proton target at $45 \mathrm{MeV}[9]$

$$
A_{h}(\overrightarrow{p p}, 45 \mathrm{MeV})=-0.795\left(\lambda_{s}^{0}+\lambda_{s}^{1}+\frac{1}{\sqrt{6}} \lambda_{s}^{2}\right) m_{N}=-(1.57 \pm 0.23) \times 10^{-7}
$$

ii) Longitudinally polarized proton scattering asymmetry from ${ }^{4} \mathrm{He}$ at $46 \mathrm{MeV}[10]$

$$
A_{h}(\vec{p} \alpha, 46 \mathrm{MeV})=-\left[0.48\left(\frac{3}{2} \lambda_{s}^{0}+\lambda_{s}^{1}\right)+1.07\left(\rho_{t}+\frac{1}{2} \lambda_{t}\right)\right] m_{N}=(3.3 \pm 0.9) \times 10^{-7}
$$


iii) Neutron spin rotation in ${ }^{4} \mathrm{He}[11]$

$$
\begin{aligned}
\frac{\mathrm{d} \phi^{n \alpha}}{\mathrm{d} z} & =\left[1.2\left(\lambda_{s}^{n n}+\frac{1}{2} \lambda_{s}^{p n}\right)-2.7\left(\rho_{t}-\frac{1}{2} \lambda_{t}\right)\right] m_{N} \mathrm{rad} / \mathrm{m} \\
& =(1.7 \pm 9.1 \pm 1.4) \times 10^{-7} \mathrm{rad} / \mathrm{m}
\end{aligned}
$$

iv) Photon asymmetry in $\vec{n} p \rightarrow d \gamma[12]$

$$
A_{\gamma}=-0.11 M_{N} \rho_{t}=(-1.2 \pm 1.9 \pm 0.2) \times 10^{-7}
$$

Unfortunately the last two experiments are not currently of a precision which is able to place realistic limits on the Danilov parameters. However, the radiative capture experiment is being continued at SNS, which should yield a strong bound.

A fifth experiment is obviously required and there are a number of possibilities, such as $\vec{n} d \rightarrow t \gamma, \vec{n}^{3} \mathrm{He} \rightarrow p t, \vec{\gamma} d \rightarrow n p$, etc. However, it is not clear at this time which of these are realistic possibilities.

On the theoretical side it is important to set up an effective field theory formalism which can be used to analyze these experiments. There are various such effective Lagrangians which have been suggested and they are all equivalent. All that is needed is a Rosetta stone to translate between them. For the purposes of this note, we write, for example, the partial wave representation, which reads [13]

$$
\begin{aligned}
& \mathcal{L}_{P V}=-\left[g^{3} S_{1}-{ }^{1} P_{1} t_{a}^{\dagger} N^{T} \sigma_{2} \tau_{2} i \nabla_{a} N\right. \\
& +g^{3} S_{1}-{ }^{3} P_{1} \epsilon^{a b c} t_{a}^{\dagger} N^{T} \sigma_{2} \sigma_{c} \tau_{2} \tau_{3} \nabla_{b} N \\
& +g_{\Delta I=0}^{1} S_{0}-{ }^{3} P_{0} s_{a}^{\dagger} N^{T} \sigma_{2} \vec{\sigma} \cdot i \vec{\nabla} \tau_{2} \tau_{a} N \\
& +g_{\Delta I=1}^{1} S_{0}{ }^{3} P_{0} s_{a}^{\dagger} \epsilon^{3 a b} N^{T} \sigma_{2} \vec{\sigma} \cdot i \vec{\nabla} \tau_{2} \tau_{b} N \\
& \left.+g_{\Delta I=2}^{{ }^{1} S_{0}-{ }^{3} P_{0}} s_{a}^{\dagger} \mathcal{I}^{a b} N^{T} \sigma_{2} \vec{\sigma} \cdot i \vec{\nabla} \tau_{2} \tau_{b} N\right] \text {, }
\end{aligned}
$$

where

$$
t_{a}=\frac{1}{\sqrt{8}} N^{T} \sigma_{2} \sigma_{a} \tau_{2} N, \quad s_{a}=\frac{1}{\sqrt{8}} N^{T} \tau_{2} \tau_{a} \sigma_{2} N, \quad \mathcal{I}^{a b}=(1,1,-2)_{\mathrm{diag}} .
$$

The connection with the Danilov parameters is then

$$
\begin{aligned}
& \lambda_{t}=-2 \sqrt{2} g^{3} S_{1}-{ }^{1} P_{1}, \quad \rho_{t}=-4 \sqrt{2} g^{3} S_{1}-{ }^{3} P_{1}, \quad \lambda_{s}^{0}=-2 \sqrt{2} g_{\Delta I=0}{ }^{1} S_{0}-{ }^{3} P_{0}
\end{aligned}
$$

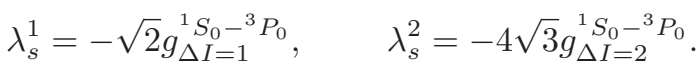

Alternative EFT formulations have been put forward by Girlanda [14] and by Zhu et al. [15] and connections between the Danilov parameters and the LECs in these Lagrangians can easily be found. (One advantage of the Zhu formalism is that connections with DDH are easily made so that estimates of the phenomenological constants can easily be given in terms of DDH best values.) 
The plan for future progress is then for experimentalists to pursue careful low energy, low A experiments which can determine (and hopefully even overdetermine) the Danilov parameters. Then these values can be mapped onto any convenient EFT formulation to give reliable values of the appropriate low energy constants. The result of a successful program in this regard would finally allow completion of the program begun over half a century ago.

This work was supported in part by the National Science Foundation under award NSF/PHY 08-55119.

\section{REFERENCES}

[1] Tanner N., Phys. Rev., 107 (1957) 1233.

[2] Krane K. S. et al., Phys. Rev. Lett., 26 (1971) 1579; Phys. Rev. C, 4 (1971) 1906.

[3] Yuan V. W. et al., Phys. Rev. C, 44 (1991) 2187; Alfimenko V. P. et al., Nucl. Phys. A, 398 (1983) 93; Masuda Y. et al., Nucl. Phys. A, 504 (1989) 269.

[4] Desplanques B., Donoghue J. F. and Holstein B. R., Ann. Phys. (N. Y.), 124 (1980) 449.

[5] Barton G., Nuovo Cimento, 19 (1961) 561.

[6] Dubovik V. M. and Zenkin S. V., Ann. Phys. (N.Y.), 172 (1986) 100.

[7] Feldman G. B., Crawford G. A., Dubach J. and Holstein B. R., Phys. Rev. C, 43 (1991) 863.

[8] Danilov G. S., Phys. Lett., 18 (1965) 40; Phys. Lett. B, 35 (1971) 579; Sov. J. Nucl. Phys., 14 (1972) 443.

[9] Balzer R. et al., Phys. Rev. Lett., 44 (1980) 699; Kystryn S. et al., Phys. Rev. Lett., 58 (1987) 1616.

[10] Lang J. et al., Phys. Rev. Lett., 54 (1985) 170.

[11] Snow W. M. et al., Phys. Rev. C, 83 (2011) 022501.

[12] Gericke M. T. et al., Phys. Rev. C, 83 (2011) 015505.

[13] Phillips D. R., Schindler M. R. and Springer R. P., Nucl. Phys. A, 822 (2009) 1.

[14] Girlanda L., Phys. Rev. C, 77 (2008) 067001.

[15] Zhu S.-L., Maekawa C. M., Holstein B. R., Ramsey-Musolf M. J. and van Kolck U., Nucl. Phys. A, 748 (2005) 435. 